\title{
Test of a Model Superconducting Magnet for the HERA ep Interaction Regions
}

\author{
B. Parker, M. Anerella, J. Escallier, A. Ghosh, A. Jain, A. Marone, J. Muratore, \\ A. Prodell, R. Thomas, P. Thompson and P. Wanderer \\ Superconducting Magnet Division, Brookhaven National Laboratory, Upton, NY 11973-5000, USA \\ H. Brueck, F. Willeke and S. Wolff \\ DESY, Notkestrasse 85, D-22607 Hamburg, Germany
}

\begin{abstract}
For the HERA luminosity upgrade two types of compact multifunction superconducting magnets, denoted GO and GG, are needed for installation inside the existing ZEUS and $H 1$ experimental detectors in the year 2000 . These magnets contain multiple concentric coil layers organized into independently powered quadrupole, dipole, skew quadrupole and skew dipole coil windings. Production of the first of three GO magnets using a newly constructed coil winding machine is currently in progress at BNL. The GG design is being completed and parallel production at BNL of three GG units will start soon. In this paper we highlight HERA upgrade magnet design challenges, present our production solutions and relate experience and results gained from warm and cold testing of short model magnets.
\end{abstract}

\section{INTRODUCTION}

The present HERA lattice has no accelerator components within the experimental region, $\pm 5.8 \mathrm{~m}$ from the interaction point (IP) but the HERA upgraded lattice [1-4] has new superconducting magnets, $G O$ and $G G$, starting at $-1.75 \mathrm{~m}$ and $+1.7 \mathrm{~m}$ to provide earlier ep beam separation and additional IP focusing. The layout is shown schematically in Fig. 1.

Both the GO and GG inner vacuum beam pipes must accommodate electron and proton beams diverging from the IP. The GG magnet needs additional horizontal aperture to pass a $25 \mathrm{~kW}$ electron beam synchrotron radiation fan generated in GO and other upstream magnets. The GO cross section is shown in Fig. 2. The GO outer cryostat is cons-

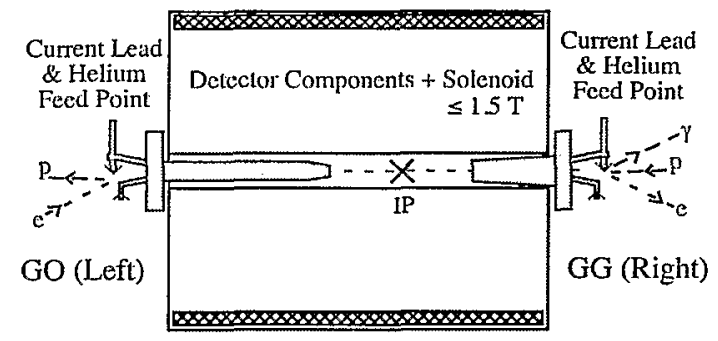

Fig. 1. Upgrade layout schematic. BNL is responsible for design and production of multilayer superconducting magnets in minicryostats, cryogenic hardware and gas-cooled lead assemblies that must fit with HERA detectors. Three magnet pairs are needed: HI, ZEUS and one spare.

Manuscript received September 27, 1999.

Work supported in part by the U.S. Department of Energy under contract No. DE-AC02-98CH10886 (BNL) trained to be less than $168 \mathrm{~mm}$ outer diameter over most of its $3.3 \mathrm{~m}$ length. About $12 \mathrm{~mm}$ radial space is available for its five cable winding layers, coil compression wraps and supercritical helium cooling space. GG radial space is so tight that we have found it necessary to taper its vacuum beam pipe, the four coil winding layers and the outer cryostat along its $1.5 \mathrm{~m}$ length. Its inner coil diameter varies from 130 to $140 \mathrm{~mm}$.

Neither magnet has enough radial space for an iron yoke. Also, as indicated in Fig. 1, the magnets are inside detector solenoids with background fields of the order of $1.5 \mathrm{~T}$; therefore, magnetic construction materials are avoided lest they spoil field quality or generate strong magnetic forces. Another solenoidal field effect is the interaction with current in the coil windings that produces magnetic forces and torques at coil ends. The cold mass will be supported against movements within the cryostat due to these forces via braces, which are stainless steel keys in G10-slots.

Finally, GO and GG are interaction region magnets, where relatively large electron beam $\beta$-functions occur. Both magnets have challenging field uniformity targets. Since the electron beam is horizontally offset $(X)$ by different amounts in GO and GG, HERA field harmonics are specified on a circle of radius $25 \mathrm{~mm}$ offset by $X=6 \mathrm{~mm}$ for $G O$ and $X=20 \mathrm{~mm}$ for $G G$. Field harmonics should be $\leq 10^{-4}$ at these offset positions. In practice, it is more convenient to measure on a circle of radius $31 \mathrm{~mm}$, centered in the magnet

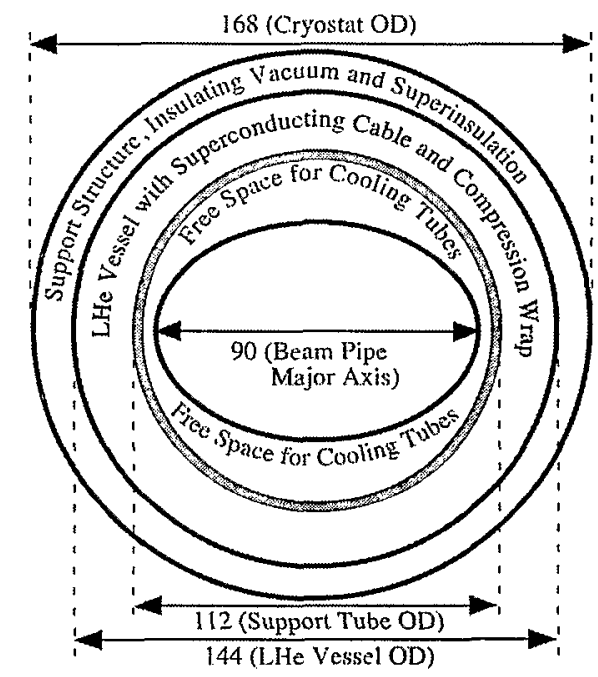

Fig. 2. GO cross section. Dimensions are given in millimeters. 
and then transform to the offset position and the smaller radius. We note that the HERA upgrade magnets must have good field regions extending slightly beyond $2 / 3$ of the inner coil radius.

\section{COIL DESIGN}

The technology used to wind the HERA upgrade magnets was originally developed for the RHIC corrector and helical magnet programs [5]. The HERA conductor is a twisted cable consisting of 7 strands of superconductor wire which are the same size as used for the cable of the helical magnets, $0.33 \mathrm{~mm}$, but having more superconductor (Cu:Sc ratio 1.8:1). The cable is made with a 6-around-1 geometry. Current ramping tests of an early prototype coil allowed us to determine that $8 \mu \mathrm{m}$ filament size is appropriate [6]. The cable is insulated with an overlapping Kapton wrap and has an added outer adhesive coating.

Through a procedure described in more detail in later sections, we build up a multilayer structure with the HERA cable. The coil patterns for each layer are generated by the BNL code COILGEN and an example of a GO quadrupole layer pattern is shown in Fig. 3. A $n$-pole layer pattern consists of $n$ sub-coils placed with appropriate symmetry, e.g. dipole, quadrupole, skew quadrupole or skew dipole.

Splicing sub-coils with the same winding handedness, i.e. always winding from inner to outer brings about a subtle field harmonic effect. Due to the way neighboring sub-coils get connected, the sub-coils differ by a fraction of a turn and thus contribute differently to the transfer function. This in turn drives the skew quadrupole harmonic in a dipole coil or a skew octopole in a quadrupole coil. Fortunately it is simple to correct for this effect by adjusting neighboring sub-coil straight section lengths by a few millimeters. The instructions for a given sub-coil pattern are saved in a COILGEN output file.

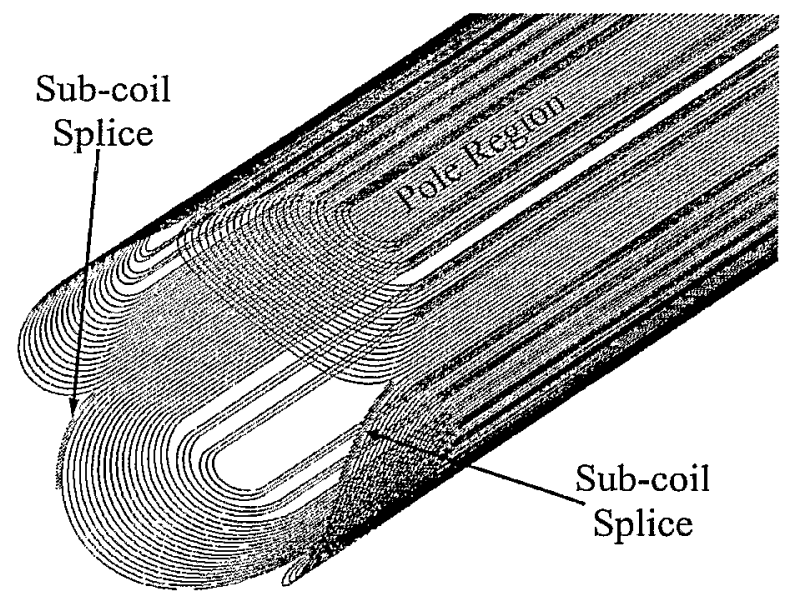

Fig. 3. GO quadrupole coil layer consisting of four independently wound sub-coils with three sub-coil splices. This coil pattern uses two straight section harmonic spacers and increased center-to-center cable end-spacing to achieve acceptable field harmonics. Recent winding tests demonstrate that we can eliminate sub-coil splices by continuously winding sub-coils in pairs. Pair winding is not implemented for GO production but should be for GG.
Typical integral field harmonics for production GO quadrupole and dipole layers are displayed in Table I. These harmonics are expressed in units at $R=31 \mathrm{~mm}$, coil centered. The marked $a_{4}$ and $a_{2}$ harmonics are brought close to zero by adjusting sub-coil straight. section lengths. Other harmonics are tuned by adjusting G10 spacer sizes and cable center-to-center spacing. Recalculating to the smaller, $25 \mathrm{~mm}$ radius circle centered at $X=6 \mathrm{~mm}$ would make high order harmonics even smaller. The harmonics are calculated directly from the files defining the patterns to the winding system. Note that we use the notation where $b_{1}=$ normal dipole, $a_{2}=$ skew quadrupole, etc. During coil optimization, undesired allowed body (2D) harmonics are adjusted to be small and then the coil ends are optimized to keep all unwanted integral harmonics as small as possible. The size and number of straight section and end spacers as well as the cable center-to-center spacing are used as optimization parameters. In general, the design values of harmonics in all the individual layers of GO and GG are well within HERA requirements. For normal quadrupole magnets, where multiple coil layers are connected in series, it is sometimes desirable to deliberately introduce additional harmonic contents in the outer layers to compensate for harmonics arising from construction errors in the inner layers. This feature has already proven to be quite useful.

\section{COIL PRODUCTION}

The coil winding for HERA is done using a new computer controlled winding machine which uses a COILGEN output file to define the winding path. The initial coil winding layer goes on a stainless steel support tube of $5 \mathrm{~mm}$ wall thickness that is prepared by wrapping first with Kapton followed by a spiral wrap of adhesive substrate. The winding machine rotates the so prepared support tube around its axis such that the cable is placed as defined by the input file. The winding

TABLE I

DESIGN INTEGRAL HARMONICS AT $\mathrm{R}=31 \mathrm{~mm}$ FOR A PRODUCTION GO QUADRUPOLE LAYER AND DIPOLE LAYER.

\begin{tabular}{cccccccc}
\hline \multicolumn{3}{c}{ GO Quadrupole Layer } & \multicolumn{5}{c}{ GO Dipole Layer } \\
\hline $\mathrm{b}_{1}$ & 0.00 & $\mathrm{a}_{1}$ & 0.00 & $\mathrm{~b}_{1}$ & 10000 & $\mathrm{a}_{1}$ & 3.1 \\
\hline $\mathrm{b}_{2}$ & 10000 & $\mathrm{a}_{2}$ & 2.3 & $\mathrm{~b}_{2}$ & 0.01 & $\mathrm{a}_{2}$ & $0.07^{*}$ \\
\hline $\mathrm{b}_{3}$ & 0.00 & $\mathrm{a}_{3}$ & 0.00 & $\mathrm{~b}_{3}$ & 0.3 & $\mathrm{a}_{3}$ & 0.1 \\
\hline $\mathrm{b}_{4}$ & 0.01 & $\mathrm{a}_{4}$ & $0.00^{*}$ & $\mathrm{~b}_{4}$ & 0.01 & $\mathrm{a}_{4}$ & -0.2 \\
\hline $\mathrm{b}_{5}$ & 0.00 & $\mathrm{a}_{5}$ & 0.00 & $\mathrm{~b}_{5}$ & 0.1 & $\mathrm{a}_{5}$ & -0.04 \\
\hline $\mathrm{b}_{6}$ & 0.04 & $\mathrm{a}_{6}$ & 0.05 & $\mathrm{~b}_{6}$ & 0.00 & $\mathrm{a}_{6}$ & -0.01 \\
\hline $\mathrm{b}_{7}$ & 0.00 & $\mathrm{a}_{7}$ & 0.00 & $\mathrm{~b}_{7}$ & -0.3 & $\mathrm{a}_{7}$ & -0.01 \\
\hline $\mathrm{b}_{8}$ & 0.00 & $\mathrm{a}_{8}$ & 0.01 & $\mathrm{~b}_{8}$ & 0.00 & $\mathrm{a}_{8}$ & 0.00 \\
\hline $\mathrm{b}_{9}$ & 0.00 & $\mathrm{a}_{9}$ & 0.00 & $\mathrm{~b}_{9}$ & -0.2 & $\mathrm{a}_{9}$ & -0.01 \\
\hline $\mathrm{b}_{10}$ & -0.1 & $\mathrm{a}_{10}$ & 0.00 & $\mathrm{~b}_{10}$ & 0.00 & $\mathrm{a}_{10}$ & 0.00 \\
\hline $\mathrm{b}_{11}$ & 0.00 & $\mathrm{a}_{11}$ & 0.00 & $\mathrm{~b}_{11}$ & 0.4 & $\mathrm{a}_{11}$ & 0.00 \\
\hline $\mathrm{b}_{12}$ & 0.00 & $\mathrm{a}_{12}$ & 0.00 & $\mathrm{~b}_{12}$ & 0.00 & $\mathrm{a}_{12}$ & 0.00 \\
\hline $\mathrm{b}_{13}$ & 0.00 & $\mathrm{a}_{13}$ & 0.00 & $\mathrm{~b}_{13}$ & -1.6 & $\mathrm{a}_{13}$ & 0.00 \\
\hline $\mathrm{b}_{14}$ & -0.4 & $\mathrm{a}_{14}$ & 0.00 & $\mathrm{~b}_{14}$ & 0.00 & $\mathrm{a}_{14}$ & 0.00 \\
\hline $\mathrm{b}_{15}$ & 0.00 & $\mathrm{a}_{15}$ & 0.00 & $\mathrm{~b}_{15}$ & 0.3 & $\mathrm{a}_{15}$ & 0.00 \\
\hline & & & & & & &
\end{tabular}


head uses a cable feed mechanism to pay out cable directly under a stylus. Ultrasonic waves, generated within the resonating stylus, are coupled through the cable into the underlying substrate. The b-stage epoxy on the substrate melts just enough to tack the cable into place on the support tube. Wiring proceeds under computer control with a gantry assembly moving the wiring head along the support tube while simultaneously tilting and rotating the winding head in a coordinated manner. There are 11 axes controlled during coil winding: 8 of the axes are mechanical and 3 are process variables.

Immediately after a complete coil layer is wound, room temperature magnetic measurements are made to verify proper cable placement. At this point the cable layer can still be removed if a severe problem is detected. The measurements are done with a rotating coil system consisting of five $0.914 \mathrm{~m}$ long tangential windings and a gravity sensor. Data are taken at both $+2 \mathrm{~A}$ and $-2 \mathrm{~A}$ excitation and background fields are subtracted using a straight line fit to the normal and skew components of each harmonic. Since all the sub-coils are not spliced together in a production configuration during the warm measurements, particular care is taken to position the leads in a reproducible manner.

If magnetic measurements confirm that the coil layer is acceptable, G1O pieces are then fitted to fill voids at the coil poles and spacers. Next, epoxy resin of type Emerson and Cuming 2850-FT, loaded with alumina, is used to fill any remaining free space between coil turns and the G10 spacers. Alumina loading imparts a low effective expansion rate and a high thermal conductivity. We use $24 \mathrm{LV}$ type hardener to lower the mix viscosity for ease of filling small voids.

To provide coil prestress, the cured coil is compression wrapped at 0.7 turns $/ \mathrm{mm}$ (18 turns/inch) and $134 \mathrm{~N}(30 \mathrm{lb})$ tension with Owens Corning S-2 glass roving which has an added B-stage epoxy impregnation [7]. The S-glass wrapped coil is cured again in a special fixture to provide a firm uniform surface for winding the next layer. In the beginning of the project, we compared coil harmonics before and after each wrap and cure step and found the field harmonics to remain constant to better than 0.5 unit. The only significant change is an anticipated small increase in transfer function as the fresh cable layer is pressed inward by the compression wrap during curing. Thus, the harmonic measurements made immediately after winding turn out to be good indicators of field quality during production.

\section{WINDING TESTS AND WARM MEASUREMENTS}

A short multilayer test magnet having three $0.61 \mathrm{~m}$ long quadrupole layers followed by an additional $0.53 \mathrm{~m}$ long dipole layer was produced using the above procedures. The magnet simulates the dipole and quadrupole layers of GO.

Warm measurements of each of the three quadrupole coils of the short test magnet, as well as with all the three coils connected in series, are summarized in Table II. Note that the first quadrupole layer has a sizeable $b_{6}$ (allowed) component. This arose because one wire had to be omitted during winding from the sub-coil pattern due to a lack of space
TABLE II

INTEGRAL HARMONICS AND INTEGRAL. TRANSFER FUNCTIONS (ITF) MEASURED WARM FOR THE TEST MAGNET QUADRUPOLE LAYERS

\begin{tabular}{|c|c|c|c|c|c|}
\hline & $\begin{array}{c}\text { Quad. } \\
\text { Layer } \\
\# 1\end{array}$ & $\begin{array}{c}\text { Quad. } \\
\text { Layer } \\
\# 2\end{array}$ & $\begin{array}{c}\text { Quad. } \\
\text { Layer } \\
\# 3\end{array}$ & $\begin{array}{c}\text { Total Meas. } \\
X=0 \mathrm{~mm} \\
\text { Rref }=31 \mathrm{~mm}\end{array}$ & $\begin{array}{l}\text { Total Meas. } \\
X=+6 \mathrm{~mm} \\
\text { Rref }=25 \mathrm{~mm}\end{array}$ \\
\hline $\operatorname{ITF}(\mathrm{T} / \mathrm{kA})$ & 5.427 & 5.049 & 4.924 & 15.408 & 15.406 \\
\hline$b_{3}$ & 6.62 & -5.47 & -8.18 & -2.03 & -1.33 \\
\hline$b_{4}$ & 1.79 & -0.63 & -0.45 & 0.25 & 0.87 \\
\hline $\mathrm{b}_{5}$ & 1.95 & -0.03 & -0.71 & 0.46 & 1.29 \\
\hline$b_{6}$ & -16.79 & 1.29 & 23.37 & 1.99 & 0.92 \\
\hline$b_{7}$ & 0.20 & 0.06 & -0.24 & 0.01 & 0.13 \\
\hline$b_{8}$ & 0.12 & 0.14 & -0.01 & 0.09 & 0.14 \\
\hline$b_{9}$ & 0.16 & 0.06 & 0.03 & 0.09 & 0.06 \\
\hline$b_{10}$ & 0.60 & 1.77 & -1.68 & 0.26 & -0.10 \\
\hline$b_{11}$ & 0.02 & -0.02 & -0.02 & 0.00 & -0.26 \\
\hline$b_{12}$ & 0.02 & 0.00 & 0.00 & 0.00 & -0.31 \\
\hline$b_{13}$ & 0.04 & 0.00 & 0.00 & 0.02 & -0.22 \\
\hline$b_{14}$ & -1.25 & -1.07 & -0.49 & -0.95 & -0.07 \\
\hline$b_{15}$ & 0.01 & 0.00 & 0.00 & 0.00 & 0.00 \\
\hline$a_{3}$ & -0.14 & -2.78 & 3.23 & -0.04 & -0.05 \\
\hline $\mathbf{a}_{4}$ & -3.80 & -2.23 & 5.55 & -0.31 & 0.06 \\
\hline$a_{5}$ & 0.54 & 0.35 & 0.26 & 0.41 & 0.17 \\
\hline$a_{6}$ & 1.17 & -0.79 & -0.94 & -0.29 & -0.15 \\
\hline$a_{7}$ & 0.02 & -0.20 & -0.05 & -0.09 & -0.01 \\
\hline$a_{8}$ & -0.03 & -0.09 & 0.01 & -0.04 & 0.01 \\
\hline$a_{9}$ & 0.19 & 0.01 & -0.03 & 0.06 & -0.04 \\
\hline
\end{tabular}

(caused by winding on a spare support tube that had a radius smaller than the design). The second layer pattern was wound with a correct average radius according to the design and had a much smaller $b_{6}$, but due to differences in radial buildup between $\mathrm{G} 10$ spacers areas and cable areas, its $b_{10}$ is slightly higher. Both layers had the same length sub-coils in each quadrant and the variation in sub-coil transfer function due to lead splicing discussed earlier was found to give a noticeable $\mathrm{a}_{4}$ term. The skew terms of order higher than the 18-pole are below 0.1 unit. Longitudinal scans indicated that the largest error harmonics come from the body (2D) and not from the coil ends

Once we measured harmonics for the first two quadrupole layers, we decided, before winding the last quadrupole layer, to see if it would be possible to compensate the observed $b_{6}$, $\mathrm{b}_{10}$ and $\mathrm{a}_{4}$ harmonics by deliberately introducing counter harmonics in the third layer. Compensation proved to be quite successful as can be seen in the last column of Table II. The largest residual harmonics, $b_{3}$ and $b_{5}$, are only 1.3 units at the $25 \mathrm{~mm}$ radius and $6 \mathrm{~mm}$ horizontal offset, even though very large corrections were made.

A single layer, $0.53 \mathrm{~m}$ long dipole coil was then wound atop the quadrupole test magnet layers described above. While the return end of the dipole coil started at the same tube location as for the quadrupole layers, at the lead end the dipole coil could not extend as far as the quadrupole did in order to be able to bring out the quadrupole leads. Also for 
field quality reasons the dipole coil end regions had to be made almost twice as long as for the quadrupole coils; so the dipole straight section ended up being very short. The measured warm harmonics for the dipole are presented in Table III for both the centered and offset reference circles. We have also carried out separate winding tests with $1.5 \mathrm{~m}$ long dipole coils, where body and end effects can be differentiated. We found $b_{2}$ and $a_{2}$ to be present in the body. The 4.5 units of $b_{3}$ probably stem from winding surface radial modulations. When wound on a round surface we see much less $b_{3}$; so the dipole layer is wound first for the GO production.

In addition to the harmonics discussed so far, layers have other significant unanticipated harmonics. Based upon subsequent winding tests we now understand that such components arise from cable placement modulation due to support tube axis offsets and bowing. For both quadrupoles and dipoles, tube non-concentricities generate harmonics of order one higher than the main field at a rate of 2 to 3 units per mil $(25 \mu \mathrm{m})$ of radial error. Corrections are incorporated in the production to counteract such errors.

\section{MOdel MAGNet COLd Testing}

Cold testing of the model magnet in a vertical dewar is presently in progress and first results are reported here. A special feature of the test setup is that we have mounted the

TABLE III

DiPOLE LAYER INTEGRAL HARMONICS AND INTEGRAL TRANSFER FUNCTION (ITF) MEASURED WARM FOR THE $0.53 \mathrm{~m}$ LONG TEST MAGNET.

\begin{tabular}{ccccc}
\hline & \multicolumn{2}{c}{$\begin{array}{c}\mathrm{X}=0 \mathrm{~mm} \\
\mathrm{Rref}=31 \mathrm{~mm}\end{array}$} & \multicolumn{2}{c}{$\begin{array}{c}\mathrm{X}=+6 \mathrm{~mm} \\
\mathrm{Rref}=25 \mathrm{~mm}\end{array}$} \\
\hline ITF $(\mathrm{Tm} / \mathrm{kA})$ & \multicolumn{2}{c}{0.19968} & \multicolumn{2}{c}{0.19966} \\
\hline $\mathrm{n}$ & $\mathrm{b}_{\mathrm{n}}$ & $\mathrm{a}_{\mathrm{n}}$ & $\mathrm{b}_{\mathrm{n}}$ & $\mathrm{a}_{\mathrm{n}}$ \\
\hline 2 & -6.75 & 2.04 & -3.32 & 1.95 \\
3 & 7.02 & -0.09 & 4.46 & 0.41 \\
4 & -0.75 & 0.99 & 0.26 & 0.81 \\
5 & 1.54 & 0.49 & 0.84 & 0.26 \\
6 & -0.09 & 0.04 & 0.31 & 0.03 \\
7 & 1.07 & -0.01 & 0.11 & -0.04 \\
8 & -0.01 & -0.03 & -0.29 & -0.03 \\
9 & -0.49 & 0.08 & -0.28 & -0.03 \\
10 & 0.00 & 0.02 & -0.21 & -0.04 \\
11 & -0.56 & 0.00 & -0.14 & -0.02 \\
12 & 0.01 & 0.00 & -0.04 & 0.00 \\
13 & -0.32 & -0.01 & 0.00 & 0.01 \\
14 & 0.00 & 0.00 & 0.02 & 0.00 \\
15 & 0.11 & 0.00 & 0.01 & 0.00 \\
\hline
\end{tabular}

model magnet inside an existing BNL superconducting solenoid in order to be able to simulate the effect of the ZEUS and $\mathrm{HI}$ detector fields on the magnet performance. In our experimental layout, the return ends of the dipole and quadrupole coils are in the central constant field region of the solenoid and the lead ends are in the fringe field outside (as will be the case for the GO and GG operation at HERA).

The present status of quench testing is as follows:

1. The quadrupole powered alone quenches at $\sim 830 \mathrm{~A}$ ( $167 \%$ of quadrupole operation).

2. For the dipole, with the quadrupole at operating current, no quenches observed up to $1000 \mathrm{~A}(300 \%$ of dipole operation).

3. For the quadrupole, with the dipole powered at $1000 \mathrm{~A}$, the quadrupole quenches at $656 \mathrm{~A}(130 \%$ of quadrupole operation). If we extrapolate to the dipole $s$ operating current, the quadrupole should quench at above $750 \mathrm{~A}$ ( $150 \%$ of quadrupole operation).

4. No changes in the quench limits were observed with the solenoid field at $1.5 \mathrm{~T}$ (maximum HERA solenoid field).

\section{CONCLUSIONS}

We can build magnets successfully with this technology. Two areas are subjects of further study and development:

1. Non-concentricities and/or out of round support assemblies. This is a mechanical problem with significant field quality implications. We are implementing correction procedures to deal with this.

2. Although all quench currents exceed the maximum operating values, they are less than the calculated short sample limits. We continue to investigate this.

\section{REFERENCES}

[1] L. Suszycki, et al., "HERA Luminosity Upgrade," DESY workshop working group report, July, 1997.

[2] W. Bartel, et al., "On Increasing HERA Collider ep IR Luminosity," Proc. EPAC 96, June, 1996.

[3] F. Willeke, "HERA Status and Future. Plans," Proc. PAC 97, Vancouver, May, 1997.

[4] M. Seidel, "Luminosity Upgrade of HERA," Proc. PAC 99, New York, March, 1999.

[5] E. Willen et al., "A Helical Magnet Design for RHIC," Proc. PAC 97, Vancouver, May, 1997.

[6] B. Parker, et al.,." Superconducting Magnets for Use Inside the HERA ep Interaction Regions," Proc. 6 th European Particle Accelerator Conference, Stockholm, June, 1998.

[7] YLA Incorporated, 2970 Bay Vista Court, Benicia, California 945100. 